\title{
Appendicular tuberculosis presenting as enterocutaneous fistula over thigh-A rare case report with review of literature
}

\author{
Vaibhav Pandey ${ }^{*}$, Ajay Narayan Gangopadhyay, Shiv Prasad Sharma, Vijayndar Kumar \\ Department of Paediatric Surgery, Institute of Medical Sciences, Banaras Hindu University, Varanasi, India; \\ *Corresponding Author: sunny.imsbhu@gmail.com \\ Received 23 July 2013; revised 11 September 2013; accepted 24 September 2013 \\ Copyright (C) 2013 Vaibhav Pandey et al. This is an open access article distributed under the Creative Commons Attribution License, \\ which permits unrestricted use, distribution, and reproduction in any medium, provided the original work is properly cited.
}

\begin{abstract}
Abdominal tuberculosis is a significant cause of morbidity and mortality in children in developing countries. Primary involvement of appendix is very rare. Presentation is usually non specific and diagnosis is made in most cases on histopathlogical examination following appendicectomy. This to our knowledge is the first case of appendicular tuberculosis presenting primarily as spontaneous enterocutaneous fistula over front of right thigh. Barium meal follow-through was diagnostic. Appendicectomy was performed followed by anti-tubercular treatment which was curative.
\end{abstract}

Keywords: Tubercular Appendicitis; Abdominal Tuberculosis; Enterocutaneous Fistula

\section{INTRODUCTION}

Abdominal tuberculosis continues to be a significant cause of morbidity and mortality in children in developing countries. Ileocaecal region is most common site of involvement and accounts for $75 \%$ of the gastrointestinal tuberculosis; primary involvement of appendix is very rare and accounts for only $0.6 \%$ to $2.9 \%$ [1]. Here we report a case of primary appendicular tuberculosis in a child presented with enterocutaneous fistula in front upper part of right thigh.

\section{CASE REPORT}

A 4-year-old female child presented with pain complaints of pain and swelling over front of right thigh associated with high grade fever. A diagnosis of a suppurative abscess was made and incision and drainage was done outside, following which she developed fecal discharge from the incision site (Figure 1). Patient had also mild pain in right iliac fossa. On examination there was guarding and mild tenderness in the right iliac fossa. Ultrasonogram of abdomen showed localized collection in the right iliac fossa. Barium meal follows through showed spillage of dye from Ileocaecal region and presence of fistulous tract towards right thigh. Exploratory laparotomy was performed, intraoperatively appendix was inflammed and there was a perforation over the tip of appendix (Figure 2) with purulent fluid in the right iliac fossa. Terminal ileum, rest of the bowel, peritoneum and other abdominal organs were normal. On further exploration, fistulous tract was found in the iliac fossa going to the front of thigh. Appendectomy followed by curettage of the tract with minimal debridement of the edge was done. Appendectomy specimen and the biopsy from the edge of the tract were sent for histopathological examination. Histological picture of the appendix showed features of tuberculosis. Investigations for pulmonary and extrapulmonary tuberculosis were negative. Anti tubercular treatment (ATT) was started and patient was discharged on 10th post operative day. On 6 months followup patient was asymptomatic with healed fistula.

\section{DISCUSSION}

Abdominal tuberculosis is a major cause of morbidity and mortality in children. Ileocaecal region is most common site with involvement in about $40 \%$ of cases. Appendicular tuberculosis is very rare as primary involvement of appendix is found only in $1 \%$ of cases [1]. The minimal contact of appendix with intestinal contents has been implicated as cause of this rare involvement. Direct haematogenous spread is the cause of isolated appendicular involvement, though some authors believe that appendicular involvement is always secondary to 


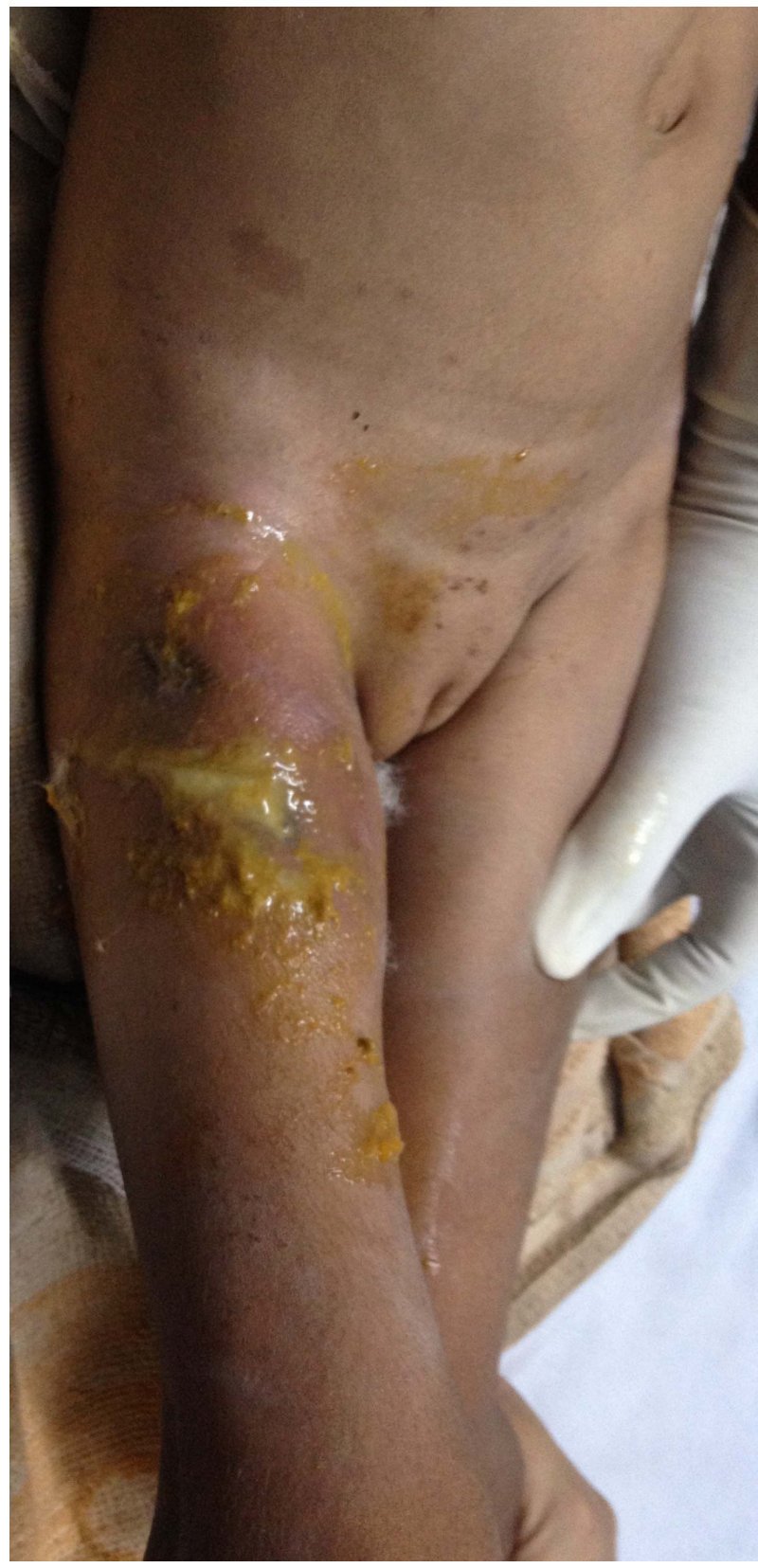

Figure 1. Enterocutaneous Fistula over front of right thigh.

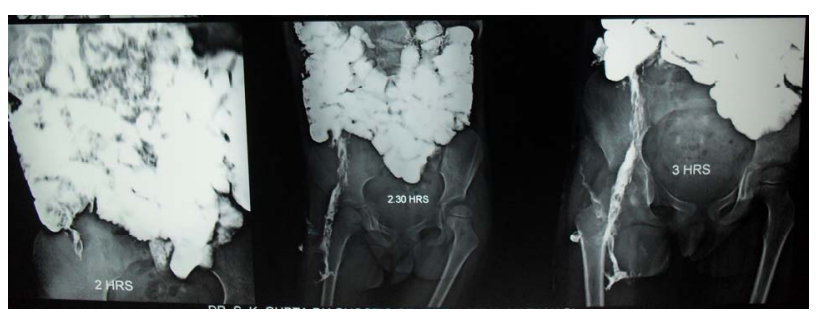

Figure 2. Barium spillage seen from ileocaecal region towards the right thigh.

primary caecal involvement [2]. In most cases diagnosis is made postoperatively following histopathlogical ex- amination. The prevalence of tuberculosis in the appendectomy specimens removed surgically for appendicitis is up to $2.9 \%$ in the reported studies [3]. In symptomatic cases recurrent episodes of right iliac fossa pain, vomiting and diarrhea are commonest presentation [4]. Though Spontaneous enterocutaneous fistula over scar of a previous appendicectomy has been reported [5], this is to our knowledge first case presenting primarily as spontaneous enterocutaneous fistula over front of right thigh. Barium studies and CECT can show the actual tract of the fistula and also locate any associated pathology [6].

In our case barium meal follow-through was able to diagnose the site of fistula preoperatively. In most cases evidence of primary tuberculosis is not found [7]. Histopathlogical examination showed lymphoid hyperplasia with associated caseating granulomas [8].

\section{CONCLUSION}

Enterocutaneous fistula on right thigh in a case of appendicitis should raise the high suspicion of underlying appendicular tuberculosis. Barium meal follow-through is very useful for preoperative diagnosis. Appendicectomy followed by anti tubercular treatment is required for cure.

\section{REFERENCES}

[1] Chou Rasheed, S., Zinicola, R., Watson, D., Bajwa, A. and McDonald, P.J. (2007) Intra-abdominal and gastrointestinal tuberculosis. Colorectal Disease, 9, 773-783. http://dx.doi.org/10.1111/j.1463-1318.2007.01337.x

[2] Singh, M.K., Arunabh and Kapoor, V.K. (1987) Tuberculosis of the appendix-A report of 17 cases and a suggested aetiopathological classification. Postgraduate Medical Journal, 63, 855-857. http://dx.doi.org/10.1136/pgmj.63.744.855

[3] Pujari, B.D., Jeyaramaiah, M. and Deodhar, S.G. (1981) Tubercular appendicitis. Journal of the Association of Physicians of India, 29, 1025-1028.

[4] Agarwal, P., Sharma, D., Agarwal, A., Agarwal, V., Tandon, A., Baghel, K.D., et al. (2004) Tuberculous appendicitis in India. Tropical Doctor, 34, 36-38.

[5] Singh, O., Gupta, S., Moses, S. and Jain, D.K. (2009) Spontaneous tubercular enterocutaneous fistula developing in the scar of a surgery done 14 years earlier. Saudi Journal of Gastroenterology, 15, 261-263. http://dx.doi.org/10.4103/1319-3767.56107

[6] Chintamani, Badran, R., Daniel, R.K., Singhal, V. and Bhatnagar, D. (2003) Spontaneous entrocutaneous fistula 27-years following radiotherapy in a patient of carcinoma penis. World Journal of Surgical Oncology, 1, 23-26. http://dx.doi.org/10.1186/1477-7819-1-23

[7] Bhansali, S.U. (1977) Abdominal tuberculosis: Experience with 300 cases. The American Journal of Gastroenterology, 67, 324-327. 
[8] Gupta, S.C., Gupta, A.K., Keswani, N.K., Singh, P.A., Tripathi, A.K. and Krishna, V. (1989) Pathology of tropi- cal appendicitis. Journal of Clinical Pathology, 42, 11691172. http://dx.doi.org/10.1136/jcp.42.11.1169 